\title{
Detection of Circulating Tumour Cells in Urothelial Cancers and Clinical Correlations: Comparison of Two Methods
}

\author{
Emanuela Fina, ${ }^{1}$ Andrea Necchi, ${ }^{2}$ Stefano Bottelli, ${ }^{3}$ Carolina Reduzzi, ${ }^{1}$ Sara Pizzamiglio, ${ }^{3}$ \\ Chiara Iacona, ${ }^{1}$ Maria Grazia Daidone, ${ }^{1}$ Paolo Verderio, ${ }^{3}$ and Vera Cappelletti ${ }^{1}$ \\ ${ }^{1}$ Department of Experimental Oncology and Molecular Medicine, Fondazione IRCCS Istituto Nazionale dei Tumori, Milano, Italy \\ ${ }^{2}$ Department of Medical Oncology, Fondazione IRCCS Istituto Nazionale dei Tumori, Milano, Italy \\ ${ }^{3}$ Unit of Medical Statistics, Biometry and Bioinformatics, Fondazione IRCCS Istituto Nazionale dei Tumori, Milano, Italy \\ Correspondence should be addressed to Paolo Verderio; paolo.verderio@istitutotumori.mi.it and \\ Vera Cappelletti; vera.cappelletti@istitutotumori.mi.it
}

Received 3 October 2016; Accepted 15 January 2017; Published 22 February 2017

Academic Editor: Marco E. M. Peluso

Copyright (c) 2017 Emanuela Fina et al. This is an open access article distributed under the Creative Commons Attribution License, which permits unrestricted use, distribution, and reproduction in any medium, provided the original work is properly cited.

Circulating tumour cells (CTC) are identified exploiting their protein/gene expression patterns or distinct size compared to blood cells. Data on CTC in bladder cancer (BC) are still scarce. We comparatively analyzed CTC enrichment by AdnaTest ProstateCancerSelect (AT) and ScreenCell ${ }^{\circledR}$ Cyto (SC) kits, combined with identification by EPCAM, MUC1, and ERBB2 expression and by cytological criteria, respectively, in 19 nonmetastatic $\left(M_{0}\right)$ and 47 metastatic $\left(M_{+}\right)$BC patients, at baseline $\left(T_{0}\right)$ and during treatment $\left(T_{1}\right)$. At $T_{0}$, CTC positivity rates by AT were higher in $M_{+}$compared to $M_{0}$ cases $(57.4 \%$ versus $25 \%, p=0.041)$. EPCAM was detected in $75 \%$ of CTC-positive samples by AT, showing increasing expression levels from $T_{0}$ to $T_{1}$ (median (interquartile range, IQR): $0.18(0.07-0.42)$ versus $0.84(0.33-1.84), p=0.005)$ in $M_{+}$cases. Overall, CTC positivity by SC was around $80 \%$ regardless of clinical setting and time point of analysis, except for a lower occurrence at $T_{1}$ in $M_{0}$ cases. At $T_{0}$, circulating tumour microemboli were more frequently ( $25 \%$ versus $8 \%$ ) detected and more numerous in $M_{+}$compared to $M_{0}$ patients. The approach used for CTC detection impacts the outcome of CTC studies. Further investigations are required to clarify the clinical validity of $\mathrm{AT}$ and SC in specific BC clinical contexts.

\section{Introduction}

Circulating tumour cells (CTC) have emerged as important blood-borne biomarkers, usable for minimally invasive tissue sampling in cancer patients, in order to track the evolution of the disease in longitudinal studies and provide additional tools potentially useful in the clinical armamentarium for personalized medicine [1]. Basically, CTC can be shed from both primary and metastatic lesions [2, 3]. Baseline CTC number proved to be correlated to disease progression in many solid tumours [2], although there are indications that CTC not merely represent a surrogate of tumour burden [4, 5]. More interestingly, as CTC display phenotypic plasticity during treatment [6], assessment of their quantitative and qualitative dynamic changes and molecular features, more than CTC count itself, might represent a promising complementary application of CTC analysis [7-9]. Indeed, CTCrelated biomarkers might be instrumental for the monitoring of spatiotemporal intratumour heterogeneity, allowing identification of CTC-specific gene signatures or genomic alterations emerging from resistant cellular clones, which might guide the choice of therapy [10, 11]. Despite this tempting scenario, the road to application for personalized medicine needs further efforts, and validation of CTC utility in clinical practice remains mandatory.

Full evaluation of CTC clinical validity and utility is essentially hampered by their rarity, heterogeneity, and plasticity $[12,13]$, three features that make CTC identification and consequently the outcome of CTC studies subject to methodological and analytical constraints and questionable 
reproducibility. In the perspective of clinical implementation, the development of a CTC-based test needs a careful standardization process $[14,15]$, but studies which compare different technical approaches are still insufficient.

At present, among the countless sophisticated technologies and bench-top devices commercially available for CTC studies, the CellSearch ${ }^{\mathrm{TM}}$ system (Janssen Diagnostic) is the only one granted FDA approval thanks to its repeatability, and it could be used as a complementary method to predict patient outcome in metastatic breast, prostate, and colorectal cancers [16, 17]. Notwithstanding the presence of CTC detected by CellSearch has been reported to be generally associated with poor prognosis in patients with solid malignancies, both at early and at advanced stages; at present no trial has yet demonstrated that modifying treatment according to CTC results provides a clinical benefit superior to the standard of care [18]. One possible reason for such a failure may be that EPCAM-based approaches (the enrichment method used in the CellSearch system) detect tumour cells which exhibit epithelial features and fail to detect subpopulations of CTC with intermediate or pure mesenchymal features $[6,19,20]$. Therefore, alternative methods allowing EPCAM-independent CTC detection, enumeration, and characterization need to be investigated.

Large interest has been recently demonstrated on the clinical significance of CTC in bladder cancer [21, 22] for which increasing incidence rates have been reported [23] and there is a pressing clinical need for new biomarkers sensitive and specific enough to parallel disease progression and treatment efficacy both in the early and in the metastatic settings. Thus, identification of patients at higher risk of relapse or progression would be beneficial for their clinical management. Data from non-CellSearch-based CTC studies are still scarce in this pathology $[21,22]$ and application of the liquid biopsy paradigm has been only recently explored [24].

On the basis of these considerations, we compared the performance of two distinct approaches in order to detect, count, and partially characterize CTC in bladder cancers. CTC analysis was carried out in parallel on the same samples using AdnaTest, a positive selection-dependent method, based on antibody-mediated recognition of surface markers followed by multiplex RT-PCR for epithelial or tumourassociated transcripts, and ScreenCell Cyto devices, a sizebased method, which exploits physical properties for CTC enrichment and cytological analysis for detection, hence unbiased with respect to biological features of tumour cells. We also provide information on the differences in CTC detection rates and fluctuations during administration of therapy, in three different clinical settings.

To our knowledge this is the first study that compares two different technical approaches to detect CTC and to explore their applicability in different clinical contexts of bladder cancer.

\section{Materials and Methods}

2.1. Case Series and Donors. Patients with bladder cancer presenting to the Department of Medical Oncology at
Fondazione IRCCS Istituto Nazionale dei Tumori (INT) between July 2012 and May 2014 were included. Three cohorts of patients were analyzed: 19 cases with muscle-invasive transitional cell carcinoma of the bladder (MIBC) receiving neoadjuvant therapy in a phase 2 trial of gemcitabine, cisplatin, and sorafenib (NCT01222676) and 47 patients who received systemic treatment for metastatic disease: 33 of whom received standard MVAC chemotherapy in the firstline setting [25], and 14 received second-line therapy in a phase 2 trial [26]. For patients with MIBC, blood samples were collected at baseline, at day 8 of each cycle of therapy. For patients with metastatic disease, blood samples were collected before starting a new line of treatment and at the beginning of each cycle of therapy.

Blood samples were collected from 39 healthy volunteers for CTC threshold definition and cell spiking studies. Patient and healthy donor characteristics are summarized in Table 1.

This study was carried out under an INT Review Boardapproved protocol allowing the collection of biological samples from patients with genitourinary cancers and written informed consent was obtained from all patients and healthy donors.

2.2. Blood Sample Collection. Samples of peripheral venous whole blood were drawn from healthy volunteers or bladder cancer patients using a $21 \mathrm{G}$ needle and collected in $4 \mathrm{~mL}$ $\mathrm{K}_{3}$ EDTA or $6 \mathrm{~mL} \mathrm{~K} \mathrm{~K}_{2}$ EDTA BD Vacutainer tubes, if intended to be processed with the AdnaTest (AdnaGen, AG, Langenhagen, Germany) or ScreenCell Cyto (ScreenCell, Sarcelles, France) kits, respectively. Samples used for CTC analysis $(5 \mathrm{~mL})$ were collected after withdrawal of blood volumes (roughly $50 \mathrm{~mL}$ ) addressed to routine tests or further clinical studies, in order to minimize the risk of contamination with epithelial skin cells during puncture. Fresh samples were stored at $4^{\circ} \mathrm{C}$ in the dark and processed within 1 hour (for AdnaTest analysis) or 2.5 hours (for ScreenCell analysis) from withdrawal.

2.3. Cell Lines and Spiking Experiments. HT-1197 and RT4 bladder cancer cell lines were purchased from ATCC (Manassas, USA) and their authenticity was verified using the STR DNA profiling with the StemElite ${ }^{\mathrm{TM}}$ ID System kit (Promega, Madison, WI, USA) by INT Genomics Core Facility. HT-1197 and RT4 cells were cultured in RPMI 1640 and McCoy's 5A media (Lonza, Slough, UK), respectively, supplemented with $10 \%$ South America origin Fetal Bovine Serum (Lonza), in humidified $5 \% \mathrm{CO}_{2}$ atmosphere.

FACS analysis for the expression of cell surface EPCAM (mouse IgG1 $\kappa$ anti- human EPCAM-PerCP-Cy7 antibody, clone 1B7, EBiosciences, San Diego, USA) and ErbB2 (mouse IgG2B anti- human ErbB2 antibody, clone 191924, R\&D Systems, Minneapolis, MN, USA) was performed on fresh monodisperse RT4 cell suspensions versus isotype control samples.

For spiking experiments, highly diluted cell suspensions were prepared in culture dishes and single viable cells (detectable by Trypan blue exclusion assay) were micropipetted under an inverted optical microscope directly into conical 
TABLE 1: Clinical and demographic characteristics for the four study groups (MIBC, metastatic patients submitted to first-line and second-line therapy and healthy donors).

\begin{tabular}{|c|c|c|c|c|}
\hline \multirow[b]{2}{*}{ Variable } & \multicolumn{4}{|c|}{ Group } \\
\hline & $\begin{array}{l}\text { Newly diagnosed, } \\
\text { receiving neoadjuvant } \\
\text { chemotherapy } \\
\text { (19 cases) }\end{array}$ & $\begin{array}{c}\text { Metastatic, receiving } \\
\text { 1st-line chemotherapy } \\
\text { (33 cases) }\end{array}$ & $\begin{array}{l}\text { Metastatic, receiving } \\
\text { 2nd-line therapy } \\
\text { (14 cases) }\end{array}$ & $\begin{array}{l}\text { Healthy } \\
\text { donors } \\
\text { (39 cases) }\end{array}$ \\
\hline \multicolumn{5}{|l|}{$\operatorname{Sex}$} \\
\hline Female & $4(21.1 \%)$ & $10(30.3 \%)$ & $1(7.1 \%)$ & $17(43.6 \%)$ \\
\hline Male & $15(78.9 \%)$ & $23(69.7 \%)$ & $13(92.9 \%)$ & $22(56.4 \%)$ \\
\hline \multicolumn{5}{|l|}{ Age } \\
\hline$<65$ & $16(84.2 \%)$ & $14(42.4 \%)$ & $6(42.9 \%)$ & $39(100 \%)$ \\
\hline$\geq 65$ & $3(15.8 \%)$ & $19(57.6 \%)$ & $8(57.1 \%)$ & 0 \\
\hline \multicolumn{5}{|l|}{ Primary tumour site } \\
\hline Bladder & $19(100 \%)$ & $28(84.8 \%)$ & $11(78.6 \%)$ & - \\
\hline Upper tract & 0 & $5(15.2 \%)$ & $3(21.4 \%)$ & - \\
\hline \multicolumn{5}{|l|}{ Metastatic sites } \\
\hline Lymph-nodes & & $22(66.7 \%)$ & $12(85.7 \%)$ & - \\
\hline Liver-lung-bone & & $13(39.4 \%)$ & $9(64.3 \%)$ & - \\
\hline Other* & & $5(15.2 \%)$ & $1(7.1 \%)$ & - \\
\hline \multicolumn{5}{|l|}{ Histology } \\
\hline Pure TCC & $2(10.5 \%)$ & $24(72.7 \%)$ & $13(92.9 \%)$ & - \\
\hline Other $^{\dagger}$ & $17(89.5 \%)$ & $7(21.2 \%)$ & $1(7.1 \%)$ & - \\
\hline \multicolumn{5}{|l|}{ Smoking habit ${ }^{\S}$} \\
\hline Never smoker & $8(42.1 \%)$ & $9(27.3 \%)$ & $2(14.3 \%)$ & $9(23.1 \%)$ \\
\hline Former smoker & $7(36.8 \%)$ & $12(36.4 \%)$ & $3(21.4 \%)$ & $5(12.8 \%)$ \\
\hline Current smoker & $4(21.1 \%)$ & $10(30.3 \%)$ & $3(21.4 \%)$ & $25(64.1 \%)$ \\
\hline
\end{tabular}

MIBC: muscle-invasive bladder cancer; TCC: transitional cell carcinoma.

${ }^{*}$ Peritoneum, bladder.

${ }^{\dagger}$ TCC + sarcomatoid, small cell differentiation, squamous cell.

${ }^{\S}$ Missing data in 2 out of 33 cases.

tubes containing $5 \mathrm{~mL}$ of whole blood from healthy volunteer. Spiked-in samples were stored at $4^{\circ} \mathrm{C}$ in the dark for no more than 1 hour before processing.

2.4. CTC Detection by Epithelial and Tumour-Associated Antigens and Multiplex-PCR. CTC enrichment by positive selection-based method was performed using the AdnaTest ProstateCancerSelect kit. Briefly, $5 \mathrm{~mL}$ of whole blood per patient was incubated with $100 \mu \mathrm{L}$ of magnetic beads, coated with antibodies against the epithelial and tumour-associated antigens EPCAM and ErbB2, on a tube rotator for 25 minutes at room temperature (RT). Cell-beads complexes were captured using the AdnaMag-L magnetic particle concentrator and washed in DPBS. Cell lysates were stored at $-20^{\circ} \mathrm{C}$ and downstream molecular analyses were performed within 2 weeks.

The expression of EPCAM, MUC1, ERBB2, CEA, and EGFR epithelial and tumour-specific markers was assessed by semiquantitative multiplex-PCR, following the manufacturer's instructions provided in the AdnaTest BreastCancerDetect and ColonCancerDetect kits. Briefly, mRNA was isolated using Dynabeads ${ }^{\circledR}$ Oligo $(\mathrm{dT})_{25}$ and retrotranscribed in a final volume of $40 \mu \mathrm{L}$, and two distinct multiplexPCR were performed using BreastCancer (EPCAM, MUC1, $E R B B 2$ and $A C T B$ ) and ColonCancer (EPCAM, CEA, EGFR, and $A C T B$ ) PrimerMixes, following each specific protocol and thermal profile. PCR products were run and solved on the Agilent 2100 Bioanalyzer (Agilent Technologies, Santa Clara, CA, USA) using the DNA 1000 kit (Agilent Technologies). The detection height threshold was set to " 0 " and markers' concentration expressed as "ng $/ \mu \mathrm{L}$ " was considered for data analysis. For quality control assessment, $A C T B$ concentration $\geq 3.0 \mathrm{ng} / \mu \mathrm{L}$ was established as necessary criterion to consider as an evaluable CTC sample, on the basis of results obtained from healthy donors with both detection kits.

2.5. CTC Detection by a Size-Based Approach. CTC isolation by size-based method was performed using the ScreenCell Cyto kit according to manufacturer's instructions. Briefly, two $2.5 \mathrm{~mL}$ aliquots of whole blood per patient were separately mixed with $4 \mathrm{~mL}$ of a proprietary red blood cell lysis and fixation buffer and incubated for 8 minutes at RT. Samples 
were filtered on two distinct isolation devices and CTC were isolated exploiting circular pores randomly distributed throughout the isolation supports (IS), that is, a microporous membrane. IS were rinsed in DPBS, air-dried, and immediately stained with Hematoxylin Solution S (Merck, Darmstadt, Germany) for 1 minute and then with Shandon Eosin Y Aqueous Solution (Thermo Fisher Scientific Inc., Waltham, MA, USA) for 30 seconds, at RT. Samples were stored at $-20^{\circ} \mathrm{C}$ until cytological evaluation by a certified pathologist.

Single CTC or circulating tumour microembolus (CTM) were identified on the basis of previously reported cytopathological criteria for malignancy [27]. Major criteria were nuclear size $\geq 20 \mu \mathrm{m}$ and nuclear-to-cytoplasmic ratio $\geq$ 0.75 , whereas irregular nuclear contours and nuclear hyperchromatism where considered among minor criteria. CTM were defined as clusters of at least two CTC (including those surrounded by platelets and fibrin), showing criteria of malignancy like those described for single CTC. Samples showing poor quality of cytology, estimated on the basis of poor preservation of the leukocytes, were excluded from the analysis. Samples were rated as CTC or CTM positive if at least 1 CTC or CTM was detected in at least one of the two IS. Results were expressed as total CTC and CTM numbers when both IS (corresponding to $5 \mathrm{~mL}$ of blood) were evaluable, according to the quality of cytology.

2.6. Statistical Analysis. The technical reliability of AdnaTest approach was assessed in ex vivo spiking experiments with bladder cancer cell lines. Different quantities (0-10-20-40) of RT4 cells $\left(99.9 \% \mathrm{EPCAM}^{+} \mathrm{ErbB}^{+}\right.$by FACS analysis) were spiked in $5 \mathrm{~mL}$ of whole blood samples and processed using AdnaTest. In parallel, the same amounts of cells were spiked in lysis buffer as a reference for capture yields evaluation and directly processed for molecular analysis. The pattern of the relationship between the concentration of $\operatorname{EPCAM}(B)$ (resulted less variable compared to $\operatorname{EPCAM}(C)$ (data not shown)), ERBB2, and EGFR markers measured from the two different extraction matrixes blood and lysis buffer was then evaluated by resorting to linear regression approaches and the adequacies of the models were assessed by $\mathrm{R}$ square index [28]. The identification the optimal cutoff for each marker was based on receiver operating characteristic (ROC) methodology by maximizing the corresponding Youden index [29]. The outcome provided by the two CTC detection approaches was dichotomized, and the degree of concordance was assessed by calculating the prevalence-adjusted and biasadjusted kappa (PABAK) statistics and its 95\% confidence interval [30] and interpreted on the basis of the Landis and Koch classification criteria [31]. The relationship between each considered continuous variable (CTC and CTM count, markers concentration) and the clinical settings $\left(M_{0}, M_{1}\right.$, and $M_{2}$ ) were investigated by mean of Kruskal-Wallis test [32]. Fisher exact test was used to assess the performance of each considered CTC detection approach according to the clinical setting.

All statistical analyses were carried out with the SAS (Version 9.2.; SAS Institute, Inc., Cary, NC) and R software by adopting a significance level of $\alpha=0.05$.

\section{Results}

3.1. Assessment of the Technical Reliability of the Two CTC Detection Approaches. As concerns the technical reliability of AdnaTest approach, we observed a linear relationship between the concentration values of each marker obtained from spiked blood samples undergoing capture and those of an equivalent number of pure RT4 cells, with $R^{2}$ indexes equal to $0.81,0.86$, and 0.96 for $E P C A M, E R B B 2$, and $E G F R$, respectively ( $M U C 1$ and $C E A$ were not detectable in RT4 cells). These results suggest that AdnaTest's efficacy in capturing tumour cells is acceptable. On the other hand, the median recovery by ScreenCell approach for 0-1-5-1025 tumour cells, spiked in $2.5 \mathrm{~mL}$ of blood, was $90 \%$ (range $0-100 \%)$. Single cell recovery was successful in 1 out of 2 independent experiments. No tumour cells were detected in healthy donors (4 individuals).

\subsection{Cutoff and Criteria for CTC Positivity Assignment by} AdnaTest. Considering that the AdnaTest approach allows indirect assessment of the presence of CTC and that baseline signals can be obtained from noncancerous cells attached to magnetic beads in nonspecific manner, positivity cutoffs were calculated by ROC curve analysis on data obtained from 39 healthy subjects and 29 patients before starting first-line chemotherapy for metastatic disease (Table 1). Compared to healthy donors, EPCAM, MUC1, and ERBB2 expression levels were higher in patients, and the areas under the ROC curves were 0.596 for EPCAM, 0.608 for MUC1, and 0.552 for ERBB2. Optimal cutoff maximizing sensitivity and specificity (Youden index) were estimated at $\geq 0.37 \mathrm{ng} / \mu \mathrm{L}$ (round off to 0.40 ) for $E P C A M, \geq 0.10 \mathrm{ng} / \mu \mathrm{L}$ for $M U C 1$, and $\geq 0.18 \mathrm{ng} / \mu \mathrm{L}$ (round off to 0.20) for ERBB2. CEA and EGFR levels were all 0 in the healthy donor group; for this reason positivity cutoff equivalent to technical detection threshold $(0.10 \mathrm{ng} / \mu \mathrm{L})$ was assigned to both markers.

As the number of positive samples in controls (healthy donors, 8/39) and cases (patients, 16/29) was similar using either combination or at least one of the three markers among EPCAM, MUC1, and ERBB2 (positivity did not change when combining aforementioned markers with CEA and EGFR), samples were considered as CTC positive when at least one among EPCAM, MUC1, and ERBB2 markers was higher than the defined cutoff value. Samples with all markers' concentration under the cutoff values were defined as CTC negative.

3.3. Analysis of CTC Positivity by AdnaTest within the Clinical Settings. At baseline, CTC positivity was lower in $M_{0}(25 \%)$ compared to $M_{+}$cases (i.e., patients under first-line therapy, hereafter referred to as " $M_{1}$," and second-line therapy, hereafter referred to as " $M_{2}$, , $57.4 \%, p=0.041$ ), with a similar trend also at $T_{1}(60 \%$ versus $86.5 \%, p=0.058)$. In $M_{0}$ cases, positivity by AdnaTest rose from 25 to $60 \%$ after the first cycle of therapy then reaching a steady state after the second cycle, whereas in $M_{+}$cases the increasing trend of CTC positivity was less apparent and mainly evident from $T_{0}$ to $T_{1}$ samples (Table 2). 
TABLE 2: CTC positivity (number of cases and percentage) by AdnaTest stratified according to clinical settings and time of analysis.

\begin{tabular}{lccc}
\hline Clinical setting & $\begin{array}{c}\text { CTC+ }(\%) \text { at baseline } \\
\left(T_{0}\right)\end{array}$ & $\begin{array}{c}\text { CTC+ (\%) after the 1st cycle } \\
\text { of therapy } \\
\left(T_{1}\right)\end{array}$ & $\begin{array}{c}\text { CTC+ }(\%) \text { after the 2nd } \\
\text { cycle of therapy } \\
\left(T_{2}\right)\end{array}$ \\
\hline$M_{0}$ & $4 / 16(25.0 \%)$ & $9 / 15(60.0 \%)$ & $10 / 16(62.5 \%)$ \\
$M_{+}$ & $27 / 47(57.4 \%)$ & $32 / 37(86.5 \%)$ & $16 / 25(64.0 \%)$ \\
$M_{1}$ & $18 / 33(54.6 \%)$ & $21 / 26(80.8 \%)$ & $16 / 25(64.0 \%)$ \\
$M_{2}$ & $9 / 14(64.3 \%)$ & $11 / 11(100 \%)$ & - \\
Total & $31 / 63(49.2 \%)$ & $41 / 52(78.8 \%)$ & $26 / 41(63.4 \%)$ \\
\hline
\end{tabular}

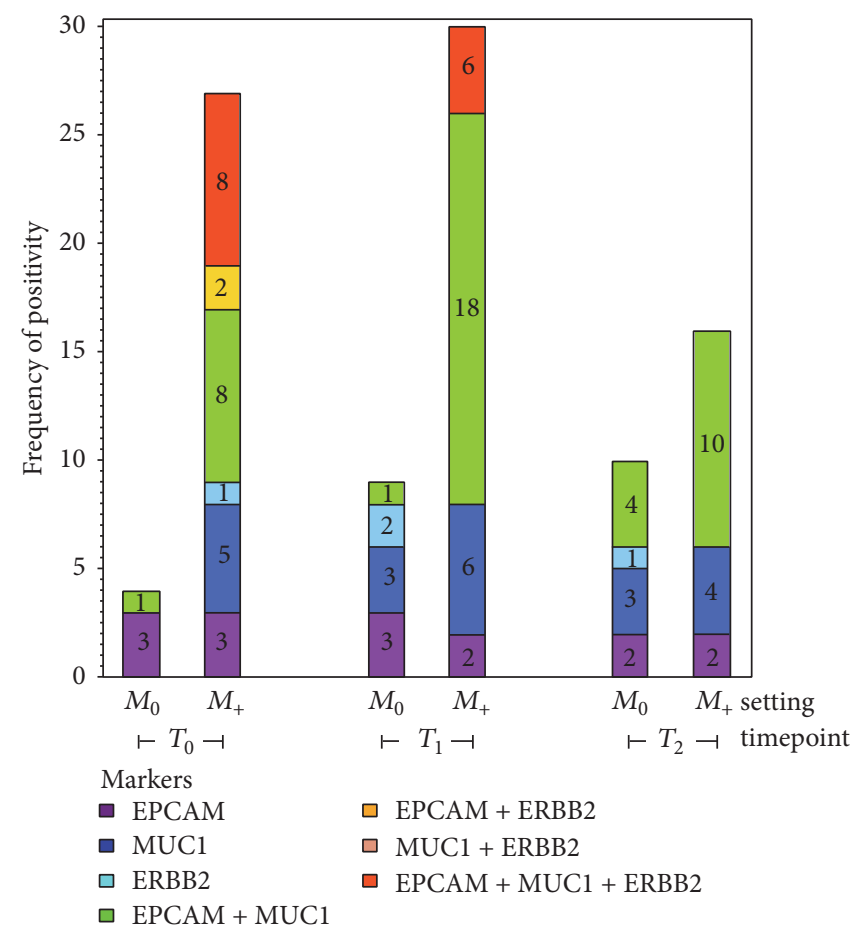

FIGURE 1: Contribution of AdnaTest markers to circulating tumour cell (CTC) positivity. The contribution of EPCAM, MUC1, ERBB2 and of all their combinations to CTC positivity is reported for $M_{0}$ and $M_{+}$cases at each time point analyzed (baseline, $T_{0}$; after first, $T_{1}$; and second, $T_{2}$, cycle of therapy). Numbers within the bars refer to the number of CTC-positive samples expressing specific markers or their combinations as indicated by the colour code.

Analysis of CTC molecular features by AdnaTest revealed a different contribution of EPCAM, MUC1, and ERBB2 markers to CTC positivity, according to the specific clinical settings and time points (Figure 1). EGFR and CEA were not considered in this analysis as they were seldom expressed. In $M_{0}$ cases EPCAM was the most represented marker in CTC+ samples (4/4 cases), and was found to be coexpressed with MUC1 in 1 out of 4 samples. In $M_{+}$cases the main contribution to CTC positivity derived from the presence of at least one marker between EPCAM and MUC1 either alone or in combination with other markers, both at baseline and during therapy. ERBB2 expression was observed in $M_{+}$ patients both at $T_{0}$ and $T_{1}$, although not as single marker, but only during therapy in $M_{0}$ patients. Interestingly, EPCAM and MUC1 were found to be more frequently coexpressed in CTC+ samples collected during therapy in $M_{+}$compared to $M_{0}$ cases. Coexpression of the three markers was never detected in $M_{0}$ cases and was instead observed in $M_{+}$cases both at baseline and at $T_{2}$.

Concentration of EPCAM, the most frequently detected marker in AdnaTest positive samples (overall 75\% at baseline), was considered as a surrogate marker of CTC burden and associated with tumour stage at different time points (Figure 2). EPCAM levels increased 4-fold from baseline to $T_{1}$ in samples from $M_{+}$patients (median for $T_{0}, 0.18$; interquantile range [IQR], 0.07-0.42 versus median for $T_{1}$, 0.84; IQR, $0.33-1.84 ; p=0.005$ ), while they did not change following neoadjuvant treatment in $M_{0}$ patients (median for $T_{0}$, 0.18; IQR, 0.09-1.01; median for $T_{1}$, 0.16; IQR, 0.06-0.47). At baseline, EPCAM levels were comparable in samples from $M_{0}$ and $M_{+}$patients (median for $M_{0}, 0.18$; IQR, 0.07-0.42; median for $M_{+}, 0.18$; IQR, 0.09-1.01). MUC1 concentrations 
TABLE 3: CTC positivity (number of cases and percentage) by ScreenCell stratified according to clinical settings and timepoints.

\begin{tabular}{lccc}
\hline Clinical setting & $\begin{array}{c}\text { CTC+ }(\%) \text { at baseline } \\
\left(T_{0}\right)\end{array}$ & $\begin{array}{c}\text { CTC+ (\%) after the 1st cycle } \\
\text { of therapy } \\
\left(T_{1}\right)\end{array}$ & $\begin{array}{c}\text { CTC+ (\%) after the 2nd } \\
\text { cycle of therapy } \\
\left(T_{2}\right)\end{array}$ \\
\hline$M_{0}$ & $11 / 13(84.6 \%)$ & $4 / 10(40.0 \%)$ & $12 / 14(85.7 \%)$ \\
$M_{+}$ & $32 / 38(84.2 \%)$ & $27 / 30(90.0 \%)$ & $16 / 17(94.1 \%)$ \\
$M_{1}$ & $22 / 27(81.5 \%)$ & $20 / 22(90.9 \%)$ & $16 / 17(94.1 \%)$ \\
$M_{2}$ & $10 / 11(90.9 \%)$ & $7 / 8(87.5 \%)$ & - \\
Total & $43 / 51(84.3 \%)$ & $31 / 40(77.5 \%)$ & $28 / 31(90.3 \%)$ \\
\hline
\end{tabular}

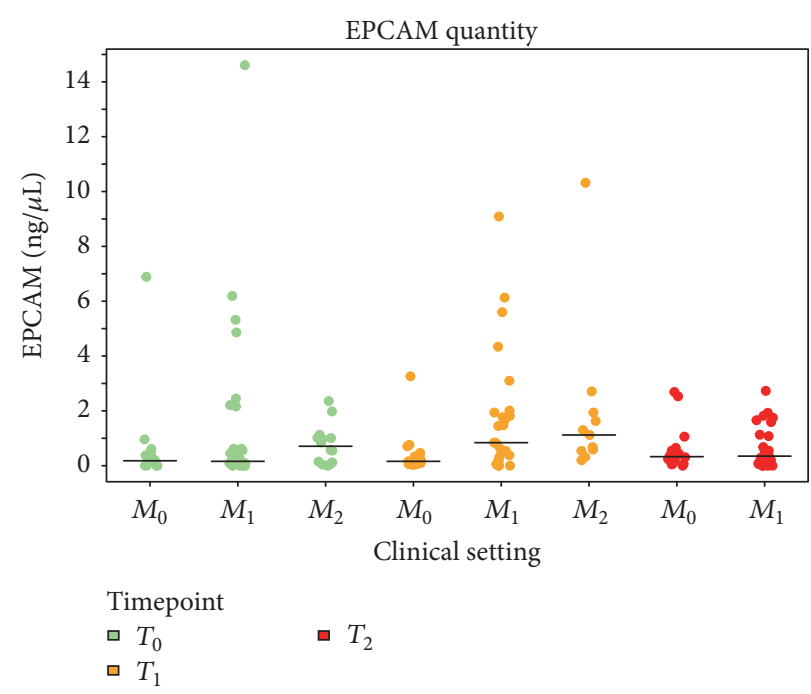

FIGURE 2: EPCAM expression in the different clinical settings. EPCAM levels (ng/ $\mu \mathrm{L})$ by RT-PCR in samples processed by AdnaTest approach are reported for each clinical setting (patients with muscle-invasive nonmetastatic bladder cancer, $M_{0}$; patients with $M_{+}$bladder cancer under first-line, $M_{1}$, or after second-line, $M_{2}$, therapy) and for each time point analyzed: baseline ( $T_{0}$, green dots), after the first ( $T_{1}$, yellow dots), and after the second $\left(T_{2}\right.$, red dots) cycle of therapy.

were similar at $T_{0}$ and $T_{1}$ in $M_{0}$ cases $(0.04(0.00-0.07)$ and $0.03(0.00-0.10))$, whereas a 5 -fold increase from $T_{0}$ to $T_{1}$ was observed in $M_{+}$cases $(0.08$ (0.05-0.28) versus 0.42 (0.19$0.73))$. ERBB2 levels were similar at any time point and in each setting, with overall median (IQR) concentrations 0.04 (0.01$0.12)$ for $T_{0}$ and $0.06(0.00-0.15)$ for $T_{1}$.

\subsection{Analysis of CTC Positivity by ScreenCell within the Clinical} Settings. Using the size-based ScreenCell approach and a positivity cutoff of at least 1 CTC or CTM, $80 \%$ of cases were defined as CTC-positive, regardless of the clinical setting and the time point of analysis, except for a lower occurrence at $T_{1}$ in $M_{0}$ cases (Table 3 ). Notwithstanding such findings, enumeration of CTC revealed no statistically significant $(p$ $=0.191$ ) lower CTC count at baseline in $M_{+}$(median 3; IQR, 1-9.5) compared to $M_{0}$ (median 9.5; IQR, 2.5-18.5) patients. Conversely, after the first cycle of therapy, CTC numbers appeared significantly ( $p=0.0004)$ increased in $M_{+}$cases (median, 8; IQR, 2-23) compared to baseline data, and a similar trend was also observed after the second cycle of therapy (median 20; IQR, 6.5-30.5; $p=0.0003$ ), while a trend toward a decrease, although not significant, was observed in
$M_{0}$ cases (median 0 ; IQR, $0-1$ at $T_{1}$ and median 1.5 ; IQR, $1-3$ at $T_{2}$ ) (Figure 3 ).

CTM counts were also considered and, despite their rarity compared to the population of single CTC (overall at $T_{0}$ they represent the 25\%, that is, 10/40 CTC-positive samples), at baseline they were more frequently detected in $M_{+}$compared to $M_{0}$ cases $(9 / 36,25 \%$ versus $1 / 12,8 \%)$ and their median and IQR numbers in CTM-positive $M_{+}$cases were 4 (2-19) compared to $1 \mathrm{CTM}$ found in the only CTM-positive $M_{0}$ case.

3.5. Concordance between AdnaTest and ScreenCell. The concordance between AdnaTest (AT) and ScreenCell (SC) in detecting CTC was assessed on matched samples belonging to the three different clinical settings collected at $T_{0}$ and $T_{1}$. As reported in Table 4, the proportion of concordant samples (AT/SC both positive or negative) was around $30 \%$ in patients with early stage disease $\left(M_{0}\right.$ setting), both at baseline and during therapy, whereas higher interassay concordances were observed in patients with metastatic disease, mainly following treatment. The overall, concordance between the two technical approaches was however poor, both at baseline (prevalence-adjusted bias-adjusted kappa, PABAK $=0.020$, 
TABLE 4: Concordance between CTC positivity evaluated by AdnaTest (AT) and Screen Cell (SC) on the same samples and as a function of the line of treatment.

\begin{tabular}{|c|c|c|c|c|c|c|c|c|}
\hline \multirow[b]{2}{*}{ Clinical setting } & \multicolumn{4}{|c|}{ Before therapy } & \multicolumn{4}{|c|}{ After therapy } \\
\hline & $N$ & $\begin{array}{c}\text { CTC+ by AT } \\
(\%)\end{array}$ & $\begin{array}{c}\text { CTC+ by SC } \\
(\%)\end{array}$ & $\begin{array}{l}\text { Concordant } \\
\text { samples (\%) }\end{array}$ & $N$ & $\begin{array}{c}\mathrm{CTC}+\text { by AT } \\
(\%)\end{array}$ & $\begin{array}{c}\text { CTC + by SC } \\
(\%)\end{array}$ & $\begin{array}{l}\text { Concordant } \\
\text { samples (\%) }\end{array}$ \\
\hline$M_{0}$ & 13 & $4(30.8)$ & $11(84.6)$ & $4(30.8 \%)$ & 10 & $5(50.0)$ & $4(40.0)$ & $3(30.0 \%)$ \\
\hline$M_{1}$ & 27 & $16(59.3)$ & $22(81.5)$ & $15(55.6 \%)$ & 21 & $17(81.0)$ & $19(90.5)$ & $15(71.4 \%)$ \\
\hline$M_{2}$ & 11 & $8(72.7)$ & $10(90.9)$ & $7(63.6 \%)$ & 8 & $8(100.0)$ & 7 (87.5) & $7(87.5 \%)$ \\
\hline Total & 51 & $28(54.9)$ & $43(84.3)$ & $26(50.9 \%)$ & 39 & $30(76.9)$ & $30(76.9)$ & $25(64.1 \%)$ \\
\hline
\end{tabular}

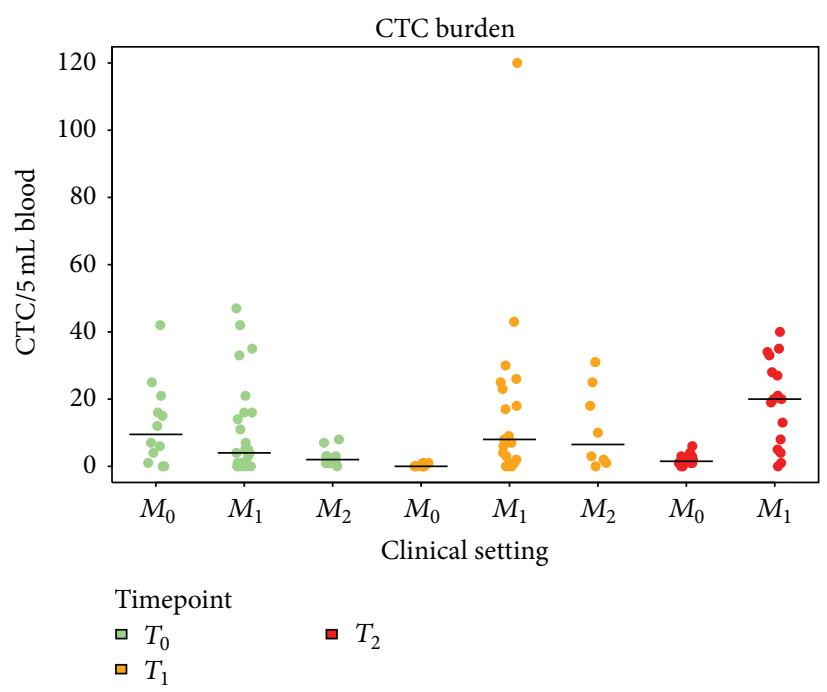

Figure 3: Circulating tumour cell (CTC) distribution in the different clinical settings. The number of CTC per $5 \mathrm{~mL}$ of blood, detected by ScreenCell approach, is reported for each clinical setting (patients with muscle-invasive nonmetastatic bladder cancer, $M_{0}$; patients with $M_{+}$ bladder cancer, under first-line, $M_{1}$, or second-line, $M_{2}$, therapy) at each time point analyzed: baseline $\left(T_{0}\right.$, green dots), after the first ( $T_{1}$, yellow dots), and after the second ( $T_{2}$, red dots) cycle of therapy.

95\% CI 0.019-0.059) and during therapy (PABAK $=0.282$, 95\% CI 0.235-0.329).

The direction of the discordance was investigated considering CTC positivity rates in samples processed by both methods (Table 4). Overall, at baseline, more samples were called positive by SC compared to AT (84.3 versus $54.9 \%$ ), with differences between $M_{0}$ and $M_{+}$(overall $M_{1}$ and $M_{2}$ ) cases (84.6 versus $30.8 \%$, and 84.2 versus $63.2 \%$, resp.). Discrepancies on CTC positivity ratings by AT and SC appeared to be also influenced by therapy administration, since it decreased after the different treatment cycles with the increase of samples called CTC-positive by AT.

\section{Discussion}

The results of this study suggest that in urothelial tumours, similarly as in other tumours [33-37], the outcome of CTC studies is markedly influenced by the specific technical approach chosen for CTC assessment. In fact, the ScreenCell approach called CTC positive more than $80 \%$ of the cases, independently from the stage of disease, whereas AdnaTest failed to detect CTC in about $40 \%$ of metastatic patients, despite systemic dissemination being expected to take place in the vast majority of cases. Moreover, in a matched comparison, its detection rate in patients with early stage disease was about 3 times lower compared to ScreenCell. The ability of AdnaTest to discriminate between healthy subjects and patients was modest (AUC around 0.60) when using EPCAM and ERBB2 as surface antigens for CTC enrichment and classical epithelial- or tumour-associated markers (EPCAM, MUC1, and ERBB2) for detection, thus providing an explanation for different CTC detection rates and scarce concordance when comparing the two methods. A possible further application could be to consider the two investigated methods in a complementary scenario by developing, for example, an algorithm based on their sequential usage in order to better characterize CTC positivity status for each patient. For AdnaTest the global contribution to CTC positivity at baseline was driven by EPCAM (19.4\%), MUC1 (16.1\%), and their combination (29.0\%), whereas the contribution of ERBB2 was not relevant considered either alone $(3.2 \%)$ or in combination with EPCAM (6.5\%). EPCAM and MUC1 were the most represented markers among CTCpositive samples especially in patients with metastatic disease, 
where positivity for $C E A$ and $E G F R$ was also observed though at low frequency. Conversely in $M_{0}$ cases only EPCAM contribution was determinant for defining CTC positivity. CTC load directly assessed either by CTC count or by considering EPCAM concentrations as surrogate marker did not appear to reflect tumour burden.

CTM were found to account for a small portion of the global CTC population, in agreement with recent observations in experimental models [38] and clinical samples [39]. Moreover, they were more frequently detected in $M_{+}$compared to $M_{0}$ cases, suggesting higher metastatic potential and possible prognostic role also in metastatic urothelial cancers, as already reported for other tumour types [36, 39-42]. Similar results have been reported by Anantharaman et al. [43] who were able to identify by cytokeratin immunostaining CTC clusters in 6/21 (28.6\%) metastatic cases and in none of four nonmetastatic bladder tumours.

As suggested by fluctuations in CTC positivity rates measured with both methods, it is possible that treatmentinduced modifications in CTC phenotypes might have impacted the test performance, and that drugs with different mechanisms of action might affect CTC dynamics, mainly when detection refers on the expression of functional markers. In $M_{0}$ cases, analysis by AdnaTest revealed an opposite trend compared to ScreenCell, as CTC positivity increased after chemotherapy for the former, whereas a stepwise decrease was observed by CTC count at each cycle of therapy. The AdnaTest result could be interpreted in different ways: it may represent an increased expression by single CTC without a change in the global CTC number, or on the opposite as an increased fraction of CTC expressing epithelial markers. We also cannot exclude the notion that chemotherapy might have triggered the release of bone marrow derived edonthelial precursors in the peripheral blood, that is, of cells that to a certain degree express epithelial markers but lose the expression of hematopoietic antigens [44]. However, such a hypothesis, although fascinating, does not seem to be supported by the observation of an increased concordance between AT and SC after chemotherapy as reported in Table 4, since bone marrow-derived precursor cells would not be identified as CTC by the morphological criteria adopted in SC. Moreover, neoadjuvant therapy before cystectomy was proved to provide clinical benefit in patients with MIBC [45], and ScreenCell-defined CTC fluctuations seem indeed to support the good treatment response. An alternative possible interpretation could be a low expression or absence of epithelial markers in CTC in nonmetastatic disease at baseline, leading to false negatives.

In patients with metastatic disease CTC positivity by AdnaTest increased after the first cycle of therapy and reached percentage value similar to baseline data after the second cycle, while CTC positivity given by their direct count was high and similar at any time point. Also in this case CTC trend was quite different and groups of distinct patients showing persistent positive or negative CTC status by AdnaTest were identified, but correlations with clinical outcome are required to understand their significance.

CTC data interpretation appears to be more straightforward in metastatic patients receiving second-line therapy, where overall CTC levels, assessed either by AdnaTest or by ScreenCell, increased after therapy administration, thus mirroring the lack of response observed in this cohort [26].

At present, excluding studies with CellSearch system, detection of CTC in patients with bladder cancer is based on the expression of different epithelial markers at mRNA level in mononuclear cells isolated from 5 to $12 \mathrm{~mL}$ of peripheral venous blood, as reported for EGFR, CK-19 and CK-20 [4651], and tumour-associated markers as MUC-7, Tenascin C, and Survivin [51, 52], with CTC positivity rates ranging from 10 to $80 \%$, according to the type of marker and the stage of disease.

Recently, the use of improved AdnaTest immune-magnetic systems also allowed the identification of CTC with stemlike features by PCR-based methods, showing that ALDH1 mainly contributed to CTC positivity compared to EPCAM, MUC1, or ERBB2 in bladder cancer patients [53].

From a biological point of view, in metastatic cases CTC are expected to revert to the epithelial phenotype through mesenchymal-to-epithelial transition process in order to colonize distant sites, whereas they should tend to maintain mesenchymal rather than epithelial features during dissemination at early stages of disease. This biological consideration might account for the discrepancy in CTC detection rates in $M_{0}$ cases observed between our AdnaTest-based approach and ScreenCell, since the latter is unbiased regarding the biological phenotype of the cell. Nevertheless, data on the role of epithelial-to-mesenchymal transition in dissemination and formation of secondary tumours are still controversial [54].

The advent of semiautomatic technologies such as the CellSearch system has allowed evaluation of the prognostic role of CTC status in patients with nonmetastatic bladder cancer [55-57], generally showing reduced disease-free survival when CTC are present, but data on the clinical role of CTC in metastatic disease are still lacking. Recent advancements in engineering and molecular biology techniques positively influenced CTC studies, which frequently entered clinical trials in the latest years. However, optimal conditions and approaches for CTC enrichment, detection, and analysis still remain to be established. Nevertheless, standardization and analytical validation steps as well as consensus-based metrics for determining limits of CTC measurement have not yet been defined at present. The number of studies addressing these issues is still limited.

\section{Conclusions}

In our study we originally developed an analytical method for CTC detection in bladder cancer, through an approach based on multiplex RT-PCR of a panel of tumour molecular markers and immunomagnetic enrichment. In addition, our study is the first one that compares the performance of two distinct CTC tests in different clinical settings of urothelial cancers, using parallel blood samples collected both at baseline and at different cycles of systemic therapy. Possible limitations of the study are represented by quantity and quality of markers chosen to capture and characterize CTC, but also by the lack of biological information on CTC isolated by filters. Both biological and technical variability could have influenced the 
test performance, since CTC might change their phenotype during hematogenous dissemination, losing some epithelial markers, and the panel of surface antigens beyond EPCAM, as ERBB2, MUC1, EGFR, and HGFR, currently exploited to identify CTC by immunological methods, might still be incomplete. On the other hand, morphological criteria are thought to be not sufficient to identify CTC [58] and in some cases the use of specific markers might help in distinguishing them from tumour-associated hematopoietic cells, as cancerassociated macrophages-like cells [59] or tumour-associated neutrophils [60]. The approaches proposed in this study require to be further investigated to analyze CTC in urothelial cancers. Whether the proposed methods will be applicable to monitor therapy response still remains to be determined in future studies.

\section{Competing Interests}

The authors declare no conflict of interests.

\section{Authors' Contributions}

Emanuela Fina, Andrea Necchi, Paolo Verderio, Vera Cappelletti, and Maria Grazia Daidone conceived and designed the experiments; Emanuela Fina, Carolina Reduzzi, and Chiara Iacona performed the experiments; Paolo Verderio, Sara Pizzamiglio, Stefano Bottelli, and Emanuela Fina analyzed the data; Emanuela Fina, Paolo Verderio, Vera Cappelletti, and Maria Grazia Daidone wrote the paper.

\section{Acknowledgments}

This work was supported in part by Italian Ministry of Health, by funds obtained through an Italian law that allows taxpayers to allocate 0.5 percent share of their income tax contribution to a research Institution of their choice and by a FIRC (Fondazione Italiana per la Ricerca sul Cancro) fellowship to Emanuela Fina. The authors thank Dr. Silvia Veneroni and the personnel of the Biobank of Fondazione IRCCS Istituto Nazionale dei Tumori (INT) for collecting and providing blood specimens, Cytometry Core Facility of INT for FACS analysis, Dr. Janine Wechsler (ScreenCell, Sarcelles, Paris) for performing cytopathological analysis, and Dr. Siegfried Hauch (AdnaGen, Langenhagen, Germany) for his technical and scientific support.

\section{References}

[1] C. Alix-Panabières and K. Pantel, "Clinical applications of circulating tumor cells and circulating tumor DNA as liquid biopsy," Cancer Discovery, vol. 6, no. 5, pp. 479-491, 2016.

[2] S. A. Joosse, T. M. Gorges, and K. Pantel, "Biology, detection, and clinical implications of circulating tumor cells," $E M B O$ Molecular Medicine, vol. 7, no. 1, pp. 1-11, 2015.

[3] K. Pantel and M. R. Speicher, "The biology of circulating tumor cells," Oncogene, vol. 35, no. 10, pp. 1216-1224, 2016.

[4] M. Cristofanilli, G. T. Budd, M. J. Ellis et al., "Circulating tumor cells, disease progression, and survival in metastatic breast cancer," New England Journal of Medicine, vol. 351, no. 8, pp. 781-791, 2004.

[5] D. A. Haber and V. E. Velculescu, "Blood-based analyses of cancer: circulating tumor cells and circulating tumor DNA," Cancer Discovery, vol. 4, no. 6, pp. 650-661, 2014.

[6] M. Yu, A. Bardia, B. S. Wittner et al., "Circulating breast tumor cells exhibit dynamic changes in epithelial and mesenchymal composition," Science, vol. 339, no. 6119, pp. 580-584, 2013.

[7] B. Mostert, A. M. Sieuwerts, J. Kraan et al., "Gene expression profiles in circulating tumor cells to predict prognosis in metastatic breast cancer patients," Annals of Oncology, vol. 26, no. 3, pp. 510-516, 2015.

[8] P. Gazzaniga, C. Raimondi, A. Gradilone et al., "Circulating tumor cells, colon cancer and bevacizumab: the meaning of zero," Annals of Oncology, vol. 22, no. 8, pp. 1929-1930, 2011.

[9] V. Musella, F. Pietrantonio, E. Di Buduo et al., "Circulating tumor cells as a longitudinal biomarker in patients with advanced chemorefractory, RAS-BRAF wild-type colorectal cancer receiving cetuximab or panitumumab," International Journal of Cancer, vol. 137, no. 6, pp. 1467-1474, 2015.

[10] A. Toss, Z. Mu, S. Fernandez, and M. Cristofanilli, "CTC enumeration and characterization: moving toward personalized medicine," Annals of Translational Medicine, vol. 2, no. 11, p. 108, 2014.

[11] M. G. Krebs, R. L. Metcalf, L. Carter, G. Brady, F. H. Blackhall, and C. Dive, "Molecular analysis of circulating tumour cellsbiology and biomarkers," Nature Reviews Clinical Oncology, vol. 11, no. 3, pp. 129-144, 2014.

[12] C. Alix-Panabières and K. Pantel, "Challenges in circulating tumour cell research," Nature Reviews Cancer, vol. 14, no. 9, pp. 623-631, 2014.

[13] M. Mego, S. A. Mani, and M. Cristofanilli, "Molecular mechanisms of metastasis in breast cancer-clinical applications," Nature Reviews Clinical Oncology, vol. 7, no. 12, pp. 693-701, 2010.

[14] D. R. Parkinson, N. Dracopoli, B. G. Petty et al., "Considerations in the development of circulating tumor cell technology for clinical use," Journal of Translational Medicine, vol. 10, no. 1, article 138, 2012.

[15] S. Bünger, M. Zimmermann, and J. K. Habermann, "Diversity of assessing circulating tumor cells (CTCs) emphasizes need for standardization: a CTC Guide to design and report trials," Cancer and Metastasis Reviews, vol. 34, no. 3, pp. 527-545, 2015.

[16] W. J. Allard, J. Matera, M. C. Miller et al., "Tumor cells circulate in the peripheral blood of all major carcinomas but not in healthy subjects or patients with nonmalignant diseases," Clinical Cancer Research, vol. 10, no. 20, pp. 6897-6904, 2004.

[17] M. C. Miller, G. V. Doyle, and L. W. M. M. Terstappen, "Significance of circulating tumor cells detected by the cellsearch system in patients with metastatic breast colorectal and prostate cancer," Journal of Oncology, vol. 2010, Article ID 617421, 8 pages, 2010.

[18] F.-C. Bidard and J.-Y. Pierga, "Clinical utility of circulating tumor cells in metastatic breast cancer," Journal of Clinical Oncology, vol. 33, no. 14, article 1622, 2015.

[19] B. Aktas, M. Tewes, T. Fehm, S. Hauch, R. Kimmig, and S. Kasimir-Bauer, "Stem cell and epithelial-mesenchymal transition markers are frequently overexpressed in circulating tumor cells of metastatic breast cancer patients," Breast Cancer Research, vol. 11, no. 4, article R46, 2009. 
[20] T. M. Gorges, I. Tinhofer, M. Drosch et al., "Circulating tumour cells escape from EpCAM-based detection due to epithelial-tomesenchymal transition," BMC Cancer, vol. 12, article 178, 2012.

[21] P. Msaouel and M. Koutsilieris, "Diagnostic value of circulating tumor cell detection in bladder and urothelial cancer: systematic review and meta-analysis," BMC Cancer, vol. 11, article 336, 2011.

[22] A. C. Small, Y. Gong, W. K. Oh, S. J. Hall, C. J. M. Van Rijn, and M. D. Galsky, "The emerging role of circulating tumor cell detection in genitourinary cancer," Journal of Urology, vol. 188, no. 1, pp. 21-26, 2012.

[23] A. Jemal, F. Bray, M. M. Center, J. Ferlay, E. Ward, and D. Forman, "Global cancer statistics," CA Cancer Journal for Clinicians, vol. 61, no. 2, pp. 69-90, 2011.

[24] G. Sonpavde, J. N. Rebecca, A. B. Apolo et al., "Circulating cell-free DNA profiling of patients with advanced urothelial carcinoma. 2016 Genitourinary Cancers Symposium," Journal of Clinical Oncology, vol. 34, supplement 2, abstract 358, 2016.

[25] A. Necchi, L. Mariani, P. Giannatempo et al., "Long-term efficacy and safety outcomes of modified (simplified) MVAC (methotrexate/vinblastine/doxorubicin/cisplatin) as frontline therapy for unresectable or metastatic urothelial cancer," Clinical Genitourinary Cancer, vol. 12, no. 3, pp. 203.el-209.el, 2014.

[26] A. Necchi, P. Giannatempo, L. Mariani et al., "PF-03446962, a fully-human monoclonal antibody against transforming growth-factor $\beta$ (TGF $\beta$ ) receptor ALK1, in pre-treated patients with urothelial cancer: an open label, single-group, phase 2 trial," Investigational New Drugs, vol. 32, no. 3, pp. 555-560, 2014.

[27] V. J. Hofman, M. I. Ilie, C. Bonnetaud et al., "Cytopathologic detection of circulating tumor cells using the isolation by size of epithelial tumor cell method: promises and pitfalls," American Journal of Clinical Pathology, vol. 135, no. 1, pp. 146-156, 2011.

[28] N. R. Draper and H. Smith, Applied Regression Analysis, John Wiley \& Sons, New York, NY, USA, 3rd edition, 1998.

[29] J. A. Hanley and B. J. McNeil, "The meaning and use of the area under a receiver operating characteristic (ROC) curve," Radiology, vol. 143, no. 1, pp. 29-36, 1982.

[30] F. K. Hoehler, "Bias and prevalence effects on kappa viewed in terms of sensitivity and specificity," Journal of Clinical Epidemiology, vol. 53, no. 5, pp. 499-503, 2000.

[31] J. R. Landis and G. G. Koch, "The measurement of observer agreement for categorical data," Biometrics, vol. 33, no. 1, pp. 159-174, 1977.

[32] M. Hollander, D. A. Wolfe, and E. Chicken, Nonparametric Statistical Methods, John Wiley \& Sons, New York, NY, USA, 2nd edition, 1999.

[33] H. K. Lin, S. Zheng, A. J. Williams et al., "Portable filter-based microdevice for detection and characterization of circulating tumor cells," Clinical Cancer Research, vol. 16, no. 20, pp. 50115018, 2010.

[34] I. Van der Auwera, D. Peeters, I. H. Benoy et al., "Circulating tumour cell detection: a direct comparison between the CellSearch System, the AdnaTest and CK-19/mammaglobin RTPCR in patients with metastatic breast cancer," British Journal of Cancer, vol. 102, no. 2, pp. 276-284, 2010.

[35] V. Müller, S. Riethdorf, B. Rack et al., "Prognostic impact of circulating tumor cells assessed with the CellSearch System and AdnaTest Breast in metastatic breast cancer patients: The DETECT Study," Breast Cancer Research, vol. 14, no. 4, article R118, 2012.
[36] J.-M. Hou, M. G. Krebs, L. Lancashire et al., "Clinical significance and molecular characteristics of circulating tumor cells and circulating tumor microemboli in patients with small-cell lung cancer," Journal of Clinical Oncology, vol. 30, no. 5, pp. 525532, 2012.

[37] E. Fina, C. Reduzzi, R. Motta et al., "Did circulating tumor cells tell us all they could? The missed circulating tumor cell message in breast cancer," International Journal of Biological Markers, vol. 30, no. 4, pp. e429-e433, 2015.

[38] N. Aceto, A. Bardia, D. T. Miyamoto et al., "Circulating tumor cell clusters are oligoclonal precursors of breast cancer metastasis," Cell, vol. 158, no. 5, pp. 1110-1122, 2014.

[39] Z. Mu, C. Wang, Z. Ye et al., "Prospective assessment of the prognostic value of circulating tumor cells and their clusters in patients with advanced-stage breast cancer," Breast Cancer Research and Treatment, vol. 154, no. 3, pp. 563-571, 2015.

[40] S. Gkountela and N. Aceto, "Stem-like features of cancer cells on their way to metastasis," Biology Direct, vol. 11, no. 1, article 33, 2016.

[41] G. Hamilton and B. Rath, "Detection of circulating tumor cells in non-small cell lung cancer," Journal of Thoracic Disease, vol. 8, no. 6, pp. 1024-1028, 2016.

[42] M.-C. Chang, Y.-T. Chang, J.-Y. Chen et al., "Clinical significance of circulating tumor microemboli as a prognostic marker in patients with pancreatic ductal adenocarcinoma," Clinical Chemistry, vol. 62, no. 3, pp. 505-513, 2016.

[43] A. Anantharaman, T. Friedlander, D. Lu et al., "Programmed death-ligand 1 (PD-L1) characterization of circulating tumor cells (CTCs) in muscle invasive and metastatic bladder cancer patients," BMC Cancer, vol. 16, no. 1, pp. 744-755, 2016.

[44] G. Fürstenberger, R. von Moos, R. Lucas et al., "Circulating endothelial cells and angiogenic serum factors during neoadjuvant chemotherapy of primary breast cancer," British Journal of Cancer, vol. 94, no. 4, pp. 524-531, 2006.

[45] M. Babjuk, W. Oosterlinck, R. Sylvester et al., "EAU guidelines on non-muscle-invasive urothelial carcinoma of the bladder, the 2011 update," European Urology, vol. 59, no. 6, pp. 997-1008, 2011.

[46] M. Kinjo, T. Okegawa, S. Horie, K. Nutahara, and E. Higashihara, "Detection of circulating MUC7-positive cells by reverse transcription-polymerase chain reaction in bladder cancer patients," International Journal of Urology, vol. 11, no. 1, pp. 38-43, 2004.

[47] P. Gazzaniga, O. Gandini, L. Giuliani et al., "Detection of epidermal growth factor receptor mRNA in peripheral blood: a new marker of circulating neoplastic cells in bladder cancer patients," Clinical Cancer Research, vol. 7, no. 3, pp. 577-583, 2001.

[48] C. J. Güdemann, J. Weitz, P. Kienle et al., "Detection of hematogenous micrometastasis in patients with transitional cell carcinoma," The Journal of Urology, vol. 164, no. 2, pp. 532-536, 2000.

[49] M. Retz, J. Lehmann, C. Röder et al., "Cytokeratin-20 reversetranscriptase polymerase chain reaction as a new tool for the detection of circulating tumor cells in peripheral blood and bone marrow of bladder cancer patients," European Urology, vol. 39, no. 5, pp. 507-517, 2001.

[50] I. Osman, M. Kang, A. Lee et al., "Detection of circulating cancer cells expressing uroplakins and epidermal growth factor receptor in bladder cancer patients," International Journal of Cancer, vol. 111, no. 6, pp. 934-939, 2004. 
[51] I. Leotsakos, P. Dimopoulos, E. Gkioka et al., "Detection of circulating tumor cells in bladder cancer using multiplex PCR assays," Anticancer Research, vol. 34, no. 12, pp. 7415-7424, 2014.

[52] P. Gazzaniga, I. Nofroni, O. Gandini et al., "Tenascin C and epidermal growth factor receptor as markers of circulating tumoral cells in bladder and colon cancer," Oncology Reports, vol. 14, no. 5, pp. 1199-1202, 2005.

[53] T. Todenhöfer, J. Hennenlotter, N. Dorner et al., “Transcripts of circulating tumor cells detected by a breast cancer-specific platform correlate with clinical stage in bladder cancer patients," Journal of Cancer Research and Clinical Oncology, vol. 142, no. 5, pp. 1013-1020, 2016.

[54] K. R. Fischer, A. Durrans, S. Lee et al., "Epithelial-tomesenchymal transition is not required for lung metastasis but contributes to chemoresistance," Nature, vol. 527, no. 7579, pp. 472-476, 2015.

[55] M. Rink, F. K. H. Chun, S. Minner et al., "Detection of circulating tumour cells in peripheral blood of patients with advanced non-metastatic bladder cancer," BJU International, vol. 107, no. 10, pp. 1668-1675, 2011.

[56] P. Gazzaniga, A. Gradilone, E. De berardinis et al., "Prognostic value of circulating tumor cells in nonmuscle invasive bladder cancer: a cell search analysis," Annals of Oncology, vol. 23, no. 9, Article ID mdr619, pp. 2352-2356, 2012.

[57] M. Rink, F. K. Chun, R. Dahlem et al., "Prognostic role and HER2 expression of circulating tumor cells in peripheral blood of patients prior to radical cystectomy: a prospective study," European Urology, vol. 61, no. 4, pp. 810-817, 2012.

[58] A. El-Heliebi, T. Kroneis, E. Zöhrer et al., "Are morphological criteria sufficient for the identification of circulating tumor cells in renal cancer?" Journal of Translational Medicine, vol. 11, article 214, 2013.

[59] D. L. Adams, S. S. Martin, R. K. Alpaugh et al., "Circulating giant macrophages as a potential biomarker of solid tumors," Proceedings of the National Academy of Sciences of the United States of America, vol. 111, no. 9, pp. 3514-3519, 2014.

[60] J. Zhang, X. Qiao, H. Shi et al., "Circulating tumor-associated neutrophils (cTAN) contribute to circulating tumor cell survival by suppressing peripheral leukocyte activation," Tumor Biology, vol. 37, no. 4, pp. 5397-5404, 2016. 


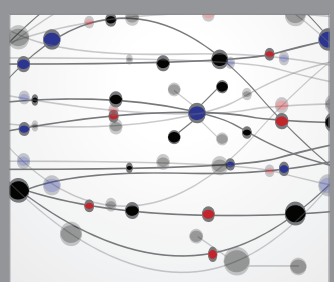

The Scientific World Journal
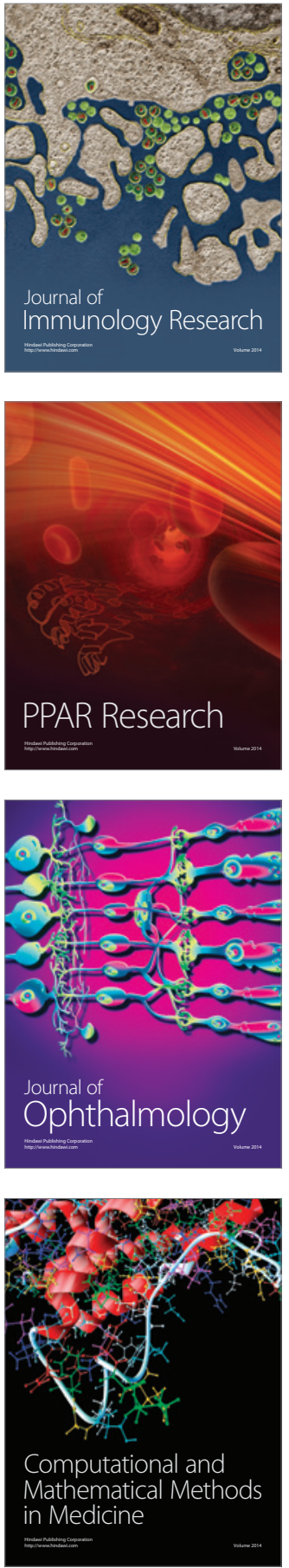

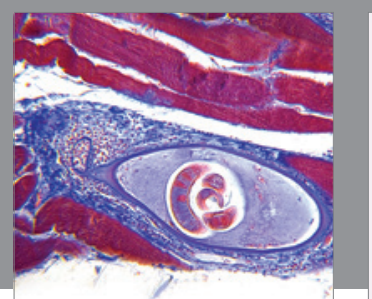

Gastroenterology Research and Practice
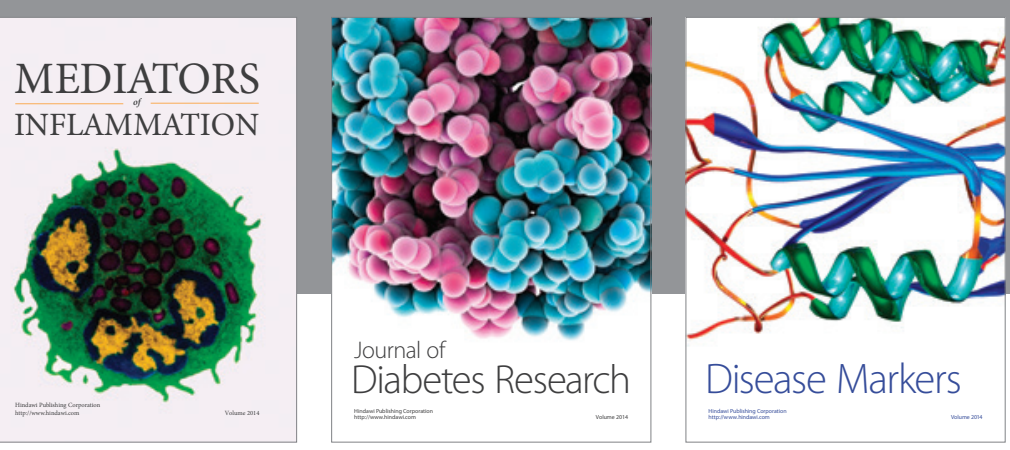

Disease Markers

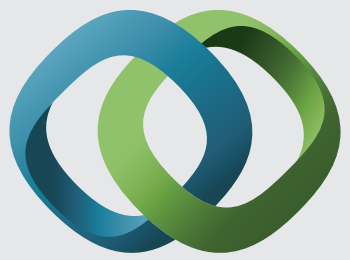

\section{Hindawi}

Submit your manuscripts at

https://www.hindawi.com
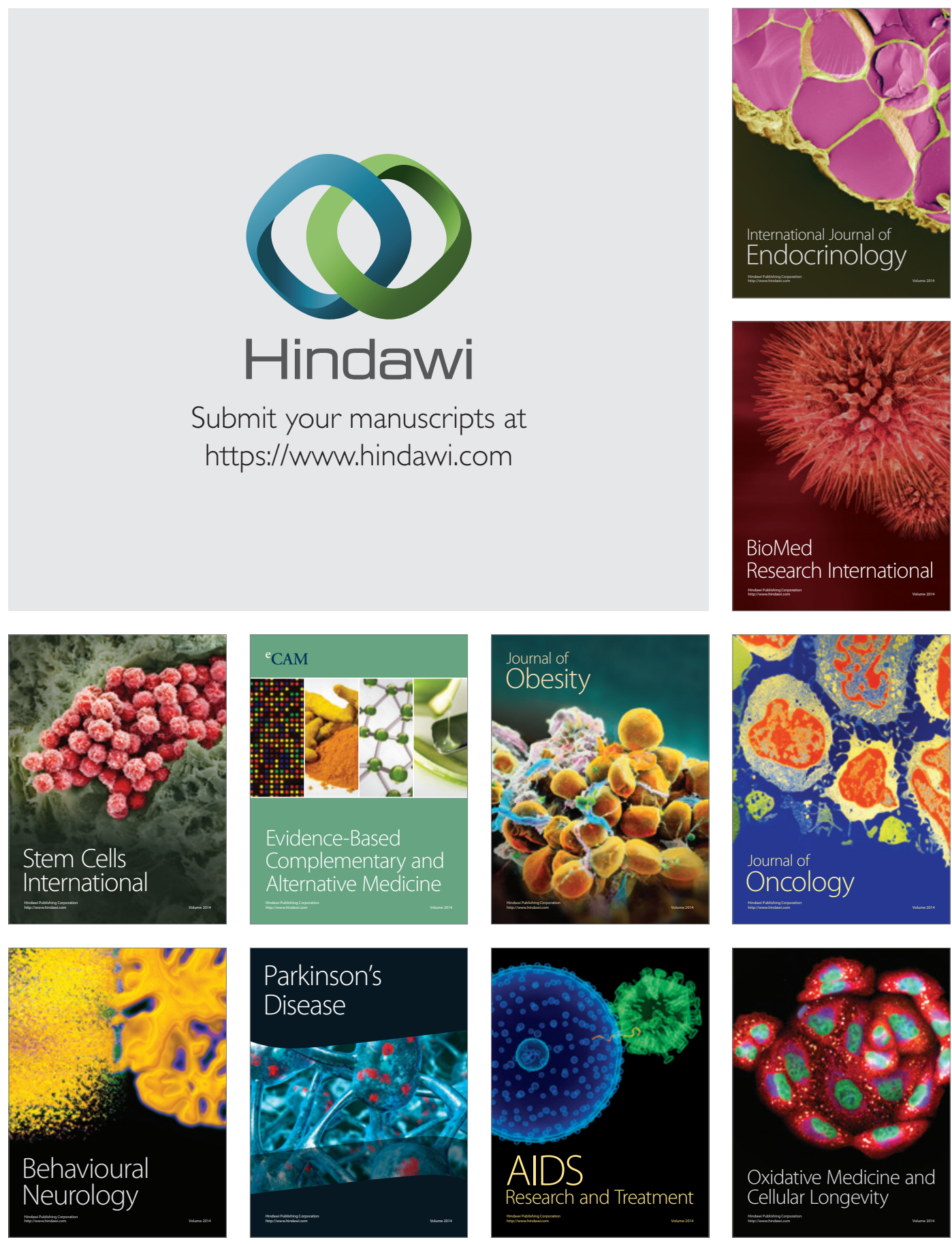